\title{
Various Types of Niche Imaging by Sonohysterography: A Pictorial Review
}

\author{
${ }^{1}$ Firoozeh Ahmadi, ${ }^{2}$ Farnaz Akhbari, ${ }^{3}$ Fatemeh Niknejad
}

\begin{abstract}
Cesarean scar defects (CSD) or niche are the myometrial discontinuity at the previous cesarean section scar region. Recently cesarean section delivery has been raised around the world markedly; therefore women with cesarean scar defects are increased and present in up to $19 \%$ of women post cesarean section. The increase of repeat cesarean section has been associated with an increase in complications in subsequent pregnancies such as scar pregnancy with life threatening bleeding, uterus rupture, placenta accreta and its subtypes and prolonged postmenstrual Spotting. The deeper the niche (or the thinner the overlying myometrium), the higher the risk for complications in a subsequent pregnancy.

Although the ability of transvaginal ultrasound (TVUS) to detect cesarean scars remains unknown, its higher frequency and proximity to the pelvic organs have been used as a powerful tool for detecting the uterine scar of a previous cesarean section.

Recently with the increasing use of sonohysterography (SHG) (transvaginal ultrasound with saline infusion) detection of scar defect has been enhanced frequently.
\end{abstract}

Keywords: Cesarean scar defect, Niche, Sonohysterography, Transvaginal ultrasound.

How to cite this article: Ahmadi F, Akhbari F, Niknejad F. Various Types of Niche Imaging by Sonohysterography: A Pictorial Review. Donald School J Ultrasound Obstet Gynecol 2014;8(3):311-315.

Source of support: Nil

Conflict of interest: None

\section{INTRODUCTION}

A uterine niche is a kind of cesarean scar defect which is defined mostly as a triangular anechoic filling defect with a depth of at least $1 \mathrm{~mm}$ in the anterior wall of the uterus usually between the uterine body and in the cervix immediately under the bladder border in the region. ${ }^{1,2}$ Dehiscence can on the site of cesarean scar occur for a variety of reasons. Influencing factors are the recurrent Cesarean sections and retroflexed uteri and catgut sutures for hysterotomy closure at previous cesarean. ${ }^{3,4}$ The associated clinical symptoms of CS defect

\footnotetext{
${ }^{1} \mathrm{MD},{ }^{2,3} \mathrm{BSc}$

${ }^{1-3}$ Department of Reproductive Imaging at Reproductive Biomedicine Research Center, Royan Institute for Reproductive Biomedicine, Academic Center for Education, Culture and Research, Tehran, Iran

Corresponding Author: Firoozeh Ahmadi, Department of Reproductive Imaging at Reproductive Biomedicine Research Center, Royan Institute, Banihashem Avenue, Hafez Street, Tehran Iran, Phone: 00989121503522 , e-mail: dr.ahmadi1390 @gmail.com
}

are: prolonged postmenstrual spotting, dysmenorrhea and chronic pelvic pain. Fertility may be affected due to presence of blood in cervical canal that impair the sperm transportation and severe defect may interfere with embryo implantation. ${ }^{5}$

Cesarean scar defect (CSD) can be identified by transvaginal sonography (TVS) ${ }^{3,6,7}$ It is not always possible to determine the number and size of scar defects or the thickness of the remaining myometrium over the defect through unenhanced ultrasound imaging. ${ }^{2,3,8}$ Saline contrast sonohysterography (SHG) may facilitate the diagnosis, especially in the measurement of myometrial thickness and the overlying intact myometrium. ${ }^{2,9,10}$

While the endometrial cavity is filled with saline, fluid outlines the niche and demarcated the border of the scar defect more clearly and facilitates detection and measurement of scar defect. These measurements are the best predictors for potential complications.

Scars are characterized as deficient if there was notable myometrial tinning on the site. The degree of thinning was expressed by ratio between a/b (Figs $1 \mathrm{~A}$ and B). The loss of more than $50 \%$ of myometrium at the scar level was arbitrarily defined as a severe deficiency ${ }^{4}$ (Fig. 2). The width of the niche also can be measured by a straight superimposed line adjoining two apposition edge of scar over the niche (c). The depth of the niche is measured between the upper tip of the niche to midpoint of this line (d) (Figs 1A and B).

Patients needed to have completely empty bladder during the test. The ideal time for sonographic evaluation is the early follicular phase, because detection of a niche and measurement of its depth and size may be better detect in thin endometrium. ${ }^{11}$

The aim of this pictorial review is to introduce varying shape of niche in our cases which is captured by SHG. The study was approved by the ethics committee of Royan institute. Written consent form was taken from all participants.

Various shapes of uterine cesarean scar defects (niche) in TVS and SHG: ${ }^{12}$

- Thin linear defect (Fig. 3)

- Wedge shape defect (Fig. 4)

- Droplet defect (Fig. 5)

- Semicircular defect (Fig. 6)

- Rectangle defect (Fig. 7)

- Inclusion cyst defect (Fig. 8) 


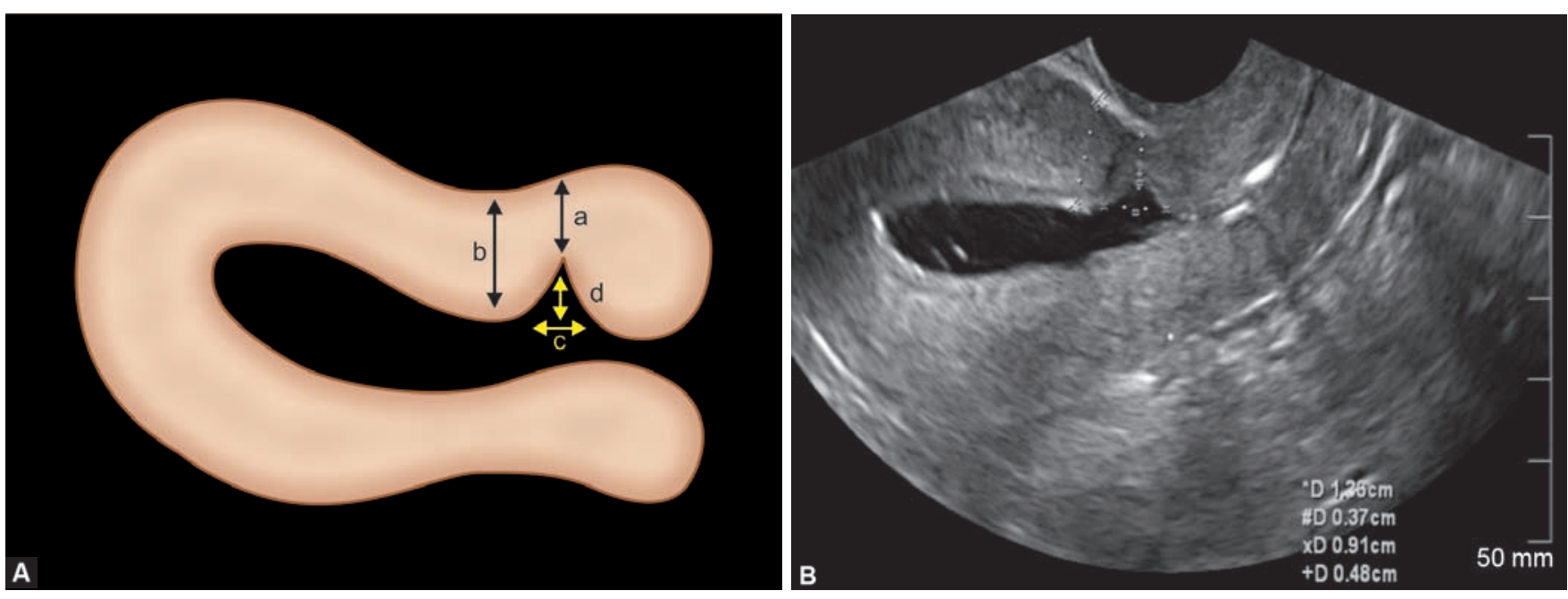

Figs $1 \mathrm{~A}$ and B: Schematic diagram illustrating measurements of thickness of the residual myometrium over the defect (a), total intact myometrium (b), width (c), and depth (d) of the cesarean scar defect (CSD). The thickness of the residual myometrium is measured between the tip of the niche and serosal surface of the uterus (a) and the thickness of the normal myometrium adjacent to the defect (b) should be recorded. The width of the niche also can be measured by a straight superimposed line adjoining two apposition edge of scar over the niche (c). The depth of the niche is measured between the upper tips of the niche to midpoint of this line (d)

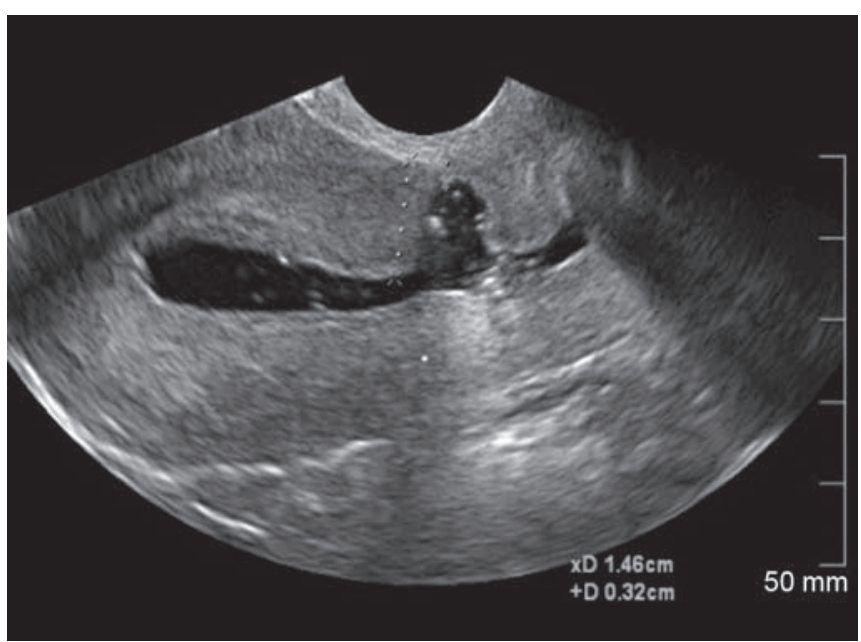

Fig. 2: Severe cesarean scar deficiency, more than $50 \%$ of myometrium at the scar level was lost

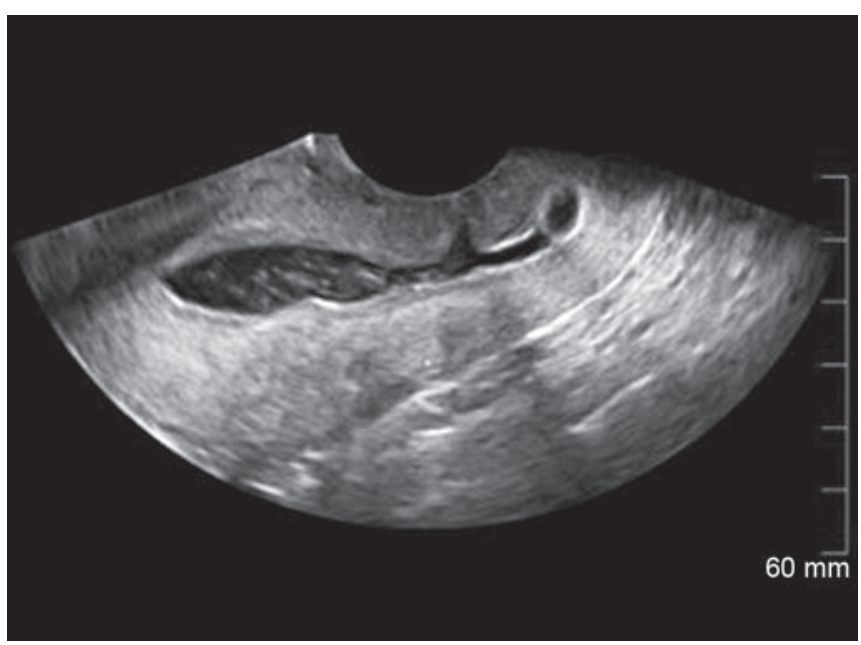

Fig. 4: Wedge shape defect (triangular)

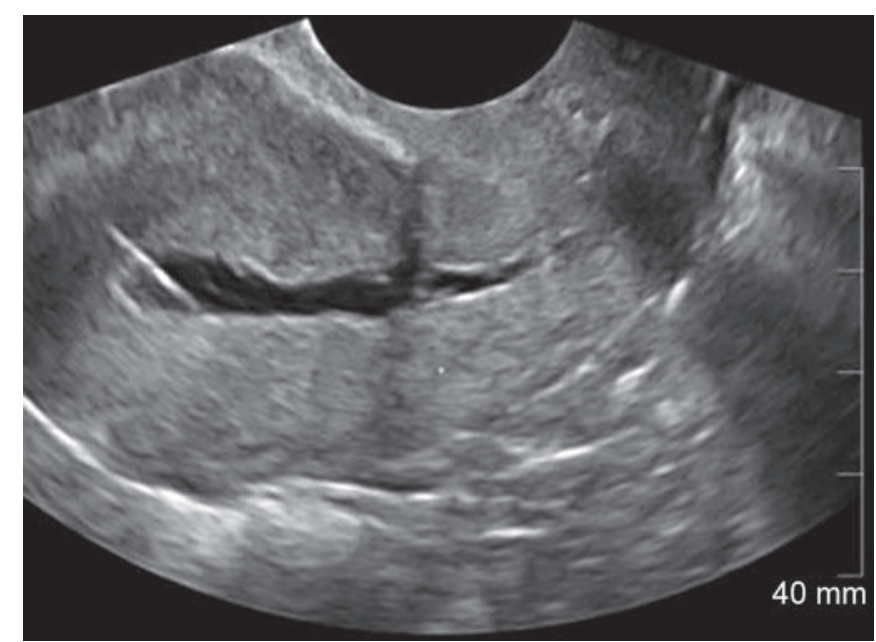

Fig. 3: Thin linear defect

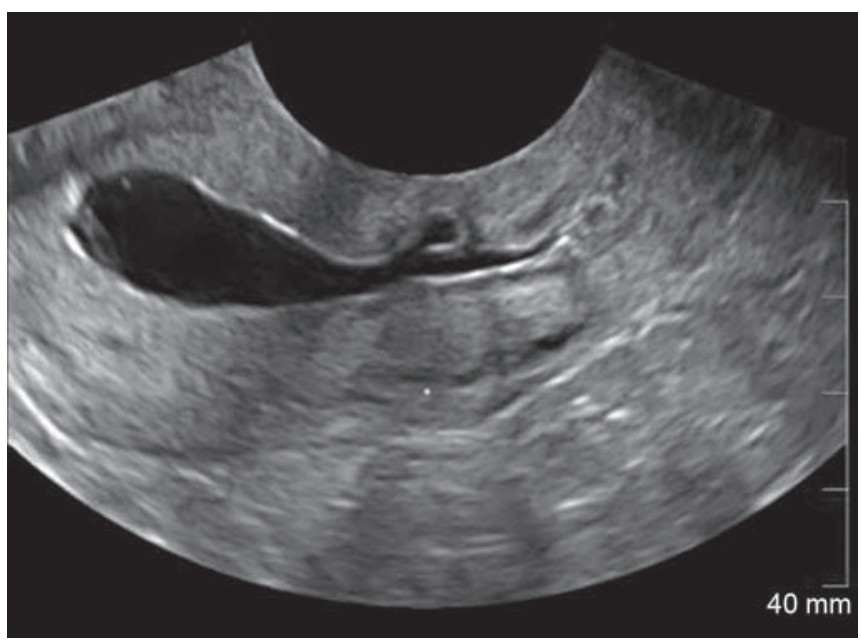

Fig. 5: Droplet 


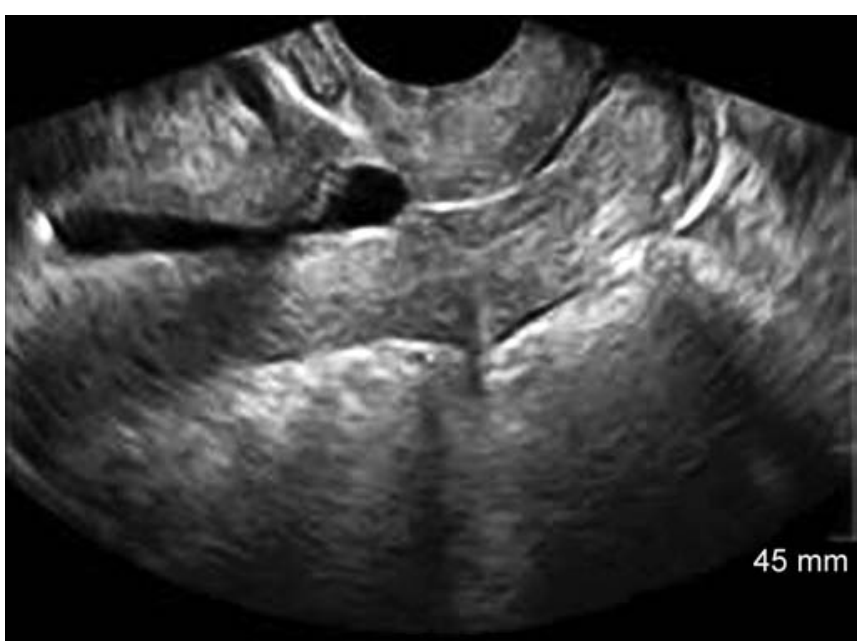

Fig. 6: Semicircular

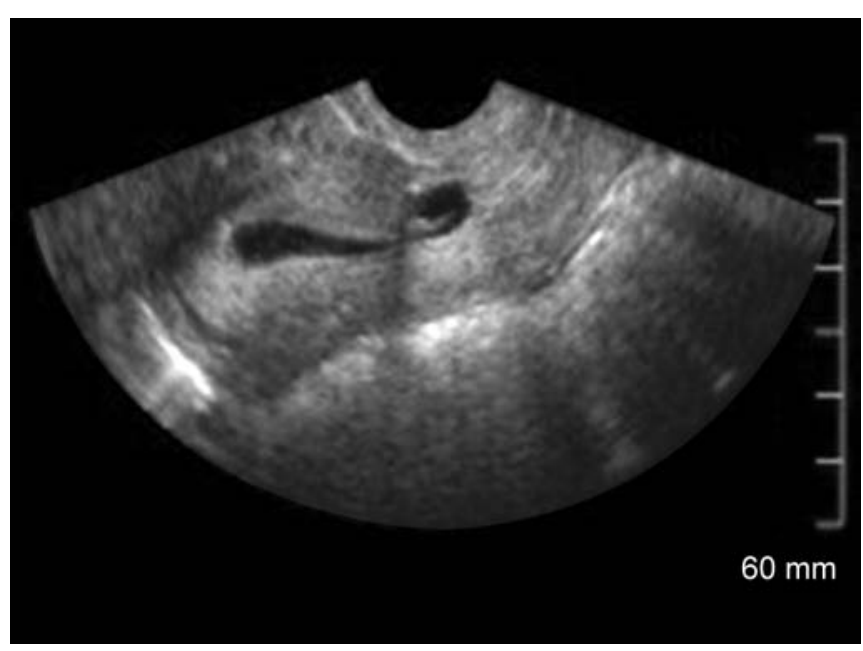

Fig. 8: Inclusion cyst

- Irregular defect (Fig. 9)

- Multiple defects (Fig. 10).

It has been reported most niches had a semicircular $(50.4 \%)$ or triangular shape $(31.6 \%))^{11}$

Comparing SHG and TVS the scar shape is similar in two techniques, but the borders of the scar defects at SHG delineate easier than in unenhanced ultrasound examination and most $\mathrm{CSD}$ appear to be larger at SHG. ${ }^{8} \mathrm{CSD}$ may be misdiagnosed with the scar for underlying pathology or normal variants such as prominent cervical glands, post myomectomy diverticulum, synechiae, and focal adenomyosis. ${ }^{13}$

According to some complications following CSD, routine sonography for detecting CSD has been suggested by some authors, in order to identify 'silent' or asymptomatic patients. ${ }^{1,3}$

\section{Some Complications Accompanied with Cesarean Scar Defect}

\section{AUB (Abnormal Uterine Bleeding) and Niche}

There is an association between the width and depth of the CSD. ${ }^{4}$ Thurmond et al articulated that AUB with niche

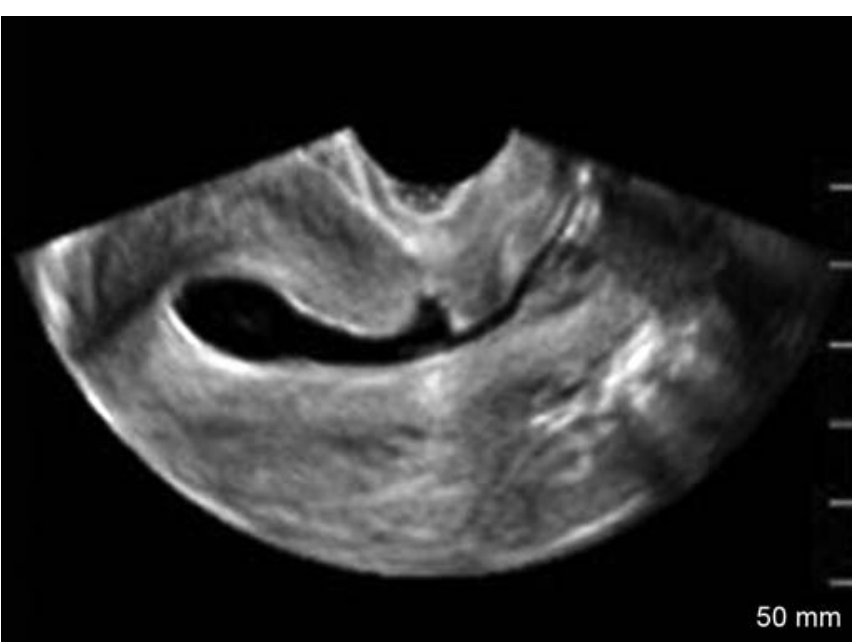

Fig. 7: Rectangle

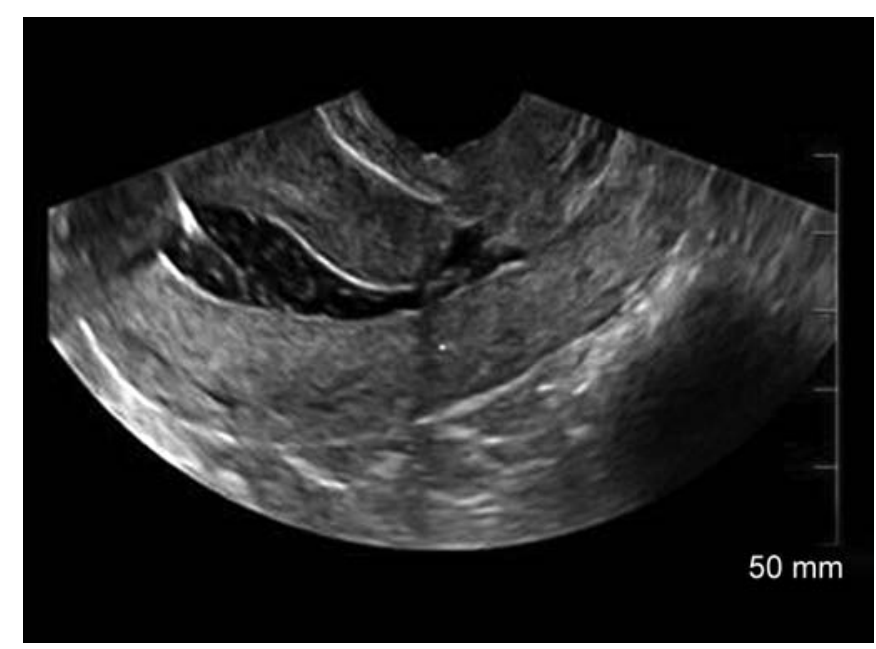

Fig. 9: Irregular

may be due to the fact that myometrial contractility around the area of the uterine scar is weak and niche acts as a reservoir. ${ }^{14}$ In recent study by Taiseer MM et al the underlying mechanisms for these symptom are discussed. ${ }^{5}$ In this study, congested endometrial fold and small polyps confined to the scar are known to be responsible for abnormal uterine bleeding (AUB) and menorrhagia.

\section{Risk of Uterine Rupture}

The presence of a niche alone probably should not be considered as a risk factor for uterine rupture during a future pregnancy. ${ }^{10}$ There is a strong association between the degree of LUS (lower uterine segment) thinning measured near term and the risk of uterine rupture at birth ${ }^{12}$ (Fig. 11). Regnard et al found that, a presence of a previous uterine scar alone should is the most important factor in determining the risk of uterine rupture, the study results showed that the frequency of defects rose as the thickness of the lower uterine segment decreased. Therefore, when the lower uterine segment was thinner than $3.5 \mathrm{~mm}$ at 37 weeks of pregnancy, the risk of uterine rupture is greater. ${ }^{10}$ 


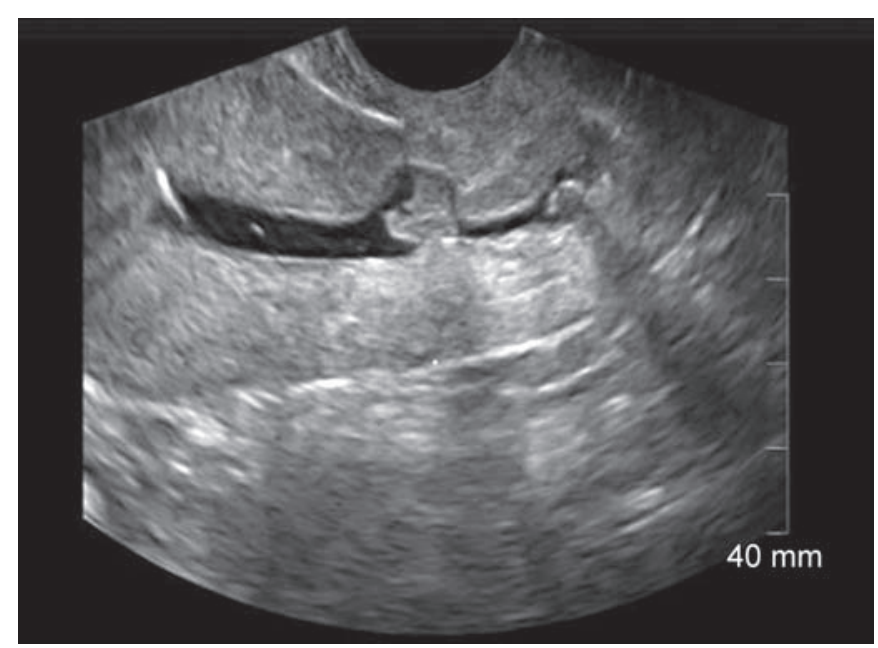

Fig. 10: Multiple (The largest defect will be measured in our study as in Vaate et al study)

\section{Cesarean Scar Pregnancy}

In CSP, the gestation sac is completely surrounded by myometrium and the fibrous tissue of the scar, quite separate from the endometrial cavity. ${ }^{2,15}$ Ultrasound is the first-line diagnostic tool for CSP. The majority of the CSPs have been diagnosed by transvaginal scan (TVS) in the early weeks of pregnancy ${ }^{2,16,17}$ (Fig. 12). Implantation of an embryo within the scar of a previous cesarean delivery is one of the rarest forms of ectopic pregnancy (EP). Such pregnancies fall into two groups; in type one the embryo starts to progress toward the uterine cavity and may result in live birth despite the high risk of a hemorrhage occurring during birth, type two consist of pregnancies in which the embryo is embedded deep within the cesarean scar (CS) and grows toward the bladder and abdominal cavity, which is very dangerous and needs to be terminated immediately.

A great deal of emphasis has been placed on its early detection and appropriate management. Early diagnosis can lead to prompt treatment, thus decreasing the likelihood of potentially serious complication. ${ }^{18,19}$

There are no financial interests that might inappropriately influence or interfere with research findings and there is no relevant financial or nonfinancial relationships in the products or services described, reviewed, evaluated or compared in this presentation.

\section{ACKNOWLEDGMENT}

The authors wish to thank imaging department staff for their assistance in figure preparation.

All the authors have participated sufficiently in the work to take public responsibility for the content.

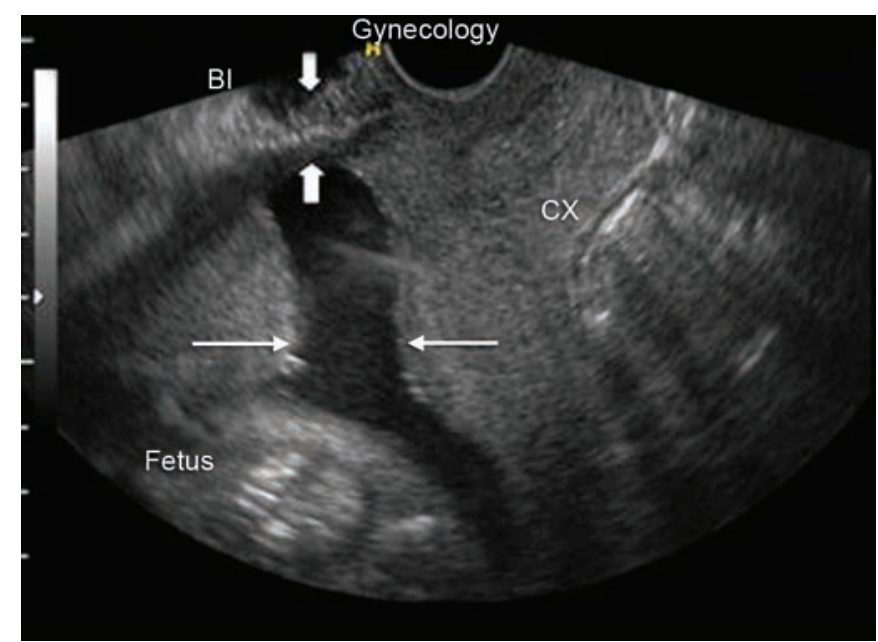

Fig. 11: Transvaginal ultrasound examination in a pregnant patient (24 weeks) demonstrated a fenestration within the uterine scar (large arrows). Thinning of the myometrium over the cesarean scar defect is seen behind the bladder (small arrows) ${ }^{17}$

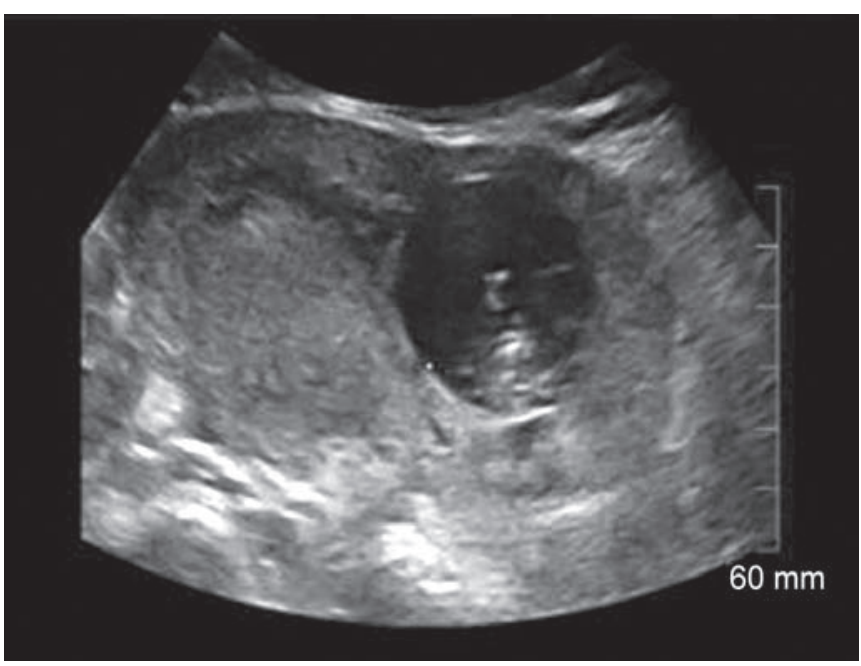

Fig 12: Transvaginal ultrasound examination shows a gestational sac (10 weeks) in the expected location of cesarean scar

\section{REFERENCES}

1. Armstrong V, Hansen WF, Van Voorhis BJ, Syrop CH. Detection of cesarean scars by transvaginal ultrasound. Obstetrics and Gynecology 2003:101.

2. Monteagudo A, Carreno C, Timor-Tritsch IE. Saline infusion sonohysterography in nonpregnant women with previous cesarean delivery. The "Niche" in the Scar. J Ultrasound Med 2001;20:1105-1115.

3. Ofili-Yebovi D, Ben-Nagi J, Sawyer E, Yazbek J, Lee C, Gonzalez J, Jurkovic D. Deficient lower-segment cesarean section scars: prevalence and risk factors. Ultrasound Obstet Gynecol 2008; 31:72-77.

4. Wang CB, Chiu WWC, Lee CY, Sun YL, Lin YH, Tseng CJ. Cesarean scar defect: correlation between Cesarean section number, defect size, clinical symptoms and uterine position. Ultrasound Obstet Gynecol 2009;34:85-89.

5. Taiseer MM, Allam NE, El Ebeissy HAEH. Caesarean section scar defects: clinical manifestations. Nature and Science 2012;10(7):11-17. 
6. Chen HY, Chen SJ, Hsieh FJ. Observation of cesarean section scar by transvaginal ultrasonography. Ultrasound Med Biol 1990; 16:443-447.

7. Osser OV, Jokubkiene L, Valentin L. High prevalence of defects in cesarean section scars at transvaginal ultrasound examination. Ultrasound Obstet Gynecol 2009;34:90-97.

8. Osser OV, Jokubkiene L, Valentin L. Cesarean section scar defects: agreement between transvaginal sonographic findings with and without saline contrast enhancement. Ultrasound Obstet Gynecol 2010;35:75-83.

9. Epstein E, Ramirez A, Skoog L, Valentin L. Transvaginal sonography, saline contrast sonohysterography and hysteroscopy for the investigation of women with postmenopausal bleeding and endometrium >5 mm. Ultrasound Obstet Gynecol 2001;18: 157-162.

10. Regnard C, Nosbusch M, Fellemans C, Benali N, Rysselberghe MV, Barlow P, Rozenberg S. Cesarean section scar evaluation by saline contrast sonohysterography. Ultrasound Obstet Gynecol 2004;23:289-292.

11. Vaate AJM BD, Lmann HAM B, Van Der Voet LF, Van Der Slikke JW, Veersema S, Huirne JAF. Ultrasound evaluation of the cesarean scar: relation between a niche and postmenstrual spotting. Ultrasound Obstet Gynecol 2011;37:93-99.
12. Jastrow N, Chaillet N, Roberge S, Morency AM, Lacasse Y, Bujold E. sonographic lower uterine segment thickness and risk of uterine scar defect: a systematic review. J Obstet Gynaecol Can 2010.

13. Surapaneni K, Silberzweig JE. Cesarean section scar diverticulum: appearance on hysterosalpingography. AJR 2008;190: 870-874.

14. Coniglio C, Dickinson JE. Pregnancy following prior Caesarean scar pregnancy rupture: lessons for modern obstetric practice. Aust NZJ Obstet Gynaecol 2004;44:1626.

15. Thurmond A, Harvey W, Smith S. Cesarean scar as a cause of abnormal vaginal bleeding: diagnosis by sonohysterography. J Ultrasound Med 1999;18:13-16.

16. Ahmadi F, Zafarani F, Haghighi H, Niknejadi M. Ectopic pregnancy in cesarean section scar: a case report. Int J Fertility and Sterility 2010:140-142.

17. Ahmadi F, Siahbazi SH, Akhbari F. Incomplete cesarean scar rupture, case report. J Reproductive Infert 2013;14(1):54.

18. Fonda J. Ultrasound diagnosis of caesarean scar defects. AJUM 2011;14(3):22-30.

19. Bignardi T, Condous G. Transrectal ultrasound-guided surgical evacuation of cesarean scar ectopic pregnancy. Ultrasound Obstet Gynecol 2010;35(4):481-485. 